\title{
Experimental demonstration of metamaterials application for mitigating scan blindness in phased array antennas
}

\author{
Pablo Rodríguez-Ulibarri ${ }^{1}$, Thomas Crépin ${ }^{2}$, Cedric Martel $^{2}$, Fabrice Boust ${ }^{2}$, Francisco Falcone ${ }^{1,3}$, \\ Claudius Loecker ${ }^{4}$, Kai Herbertz ${ }^{4}$, Thomas Bertuch ${ }^{4}$, Thierry Dousset ${ }^{5}$, Jean-Paul Martinaud ${ }^{5}$, \\ Stefano Maci $^{6}$, Jose Antonio Marcotegui ${ }^{7}$, and Miguel Beruete ${ }^{1,3, *}$ \\ 1 Department of Electrical and Electronical Engineering, Universidad Pública de Navarra, Campus Arrosadía, 31006 Pamplona, Spain \\ 2 ONERA - The French Aerospace Lab, Toulouse, France \\ 3 Institute of Smart Cities, Universidad Pública de Navarra, Campus Arrosadía, 31006 Pamplona, Spain \\ 4 Fraunhofer Institute for High Frequency Physics and Radar Techniques FHR, Wachtberg, Germany \\ 5 Thales Systèmes Aéroportés (TSA), Elancourt Cedex, France \\ 6 Department of Information Engineering and Mathematics, University of Siena, Italy \\ 7 TAFCO Metawireless S.L., Aizoáin, Spain
}

Received 10 April 2016 / Accepted 1 July 2016

\begin{abstract}
This paper presents two metamaterial-inspired solutions to mitigate the scan blindness effects in a phased array antenna. In the first solution, portions of a bed of nails are introduced in the radome to prevent the excitation of surface waves. In the second solution, a superstrate metasurface is designed to synthesize a permittivity tensor optimized to achieve a wide angle impedance matching. In both approaches, the numerical simulations are successfully compared with measurements of a phased array antenna prototype with 100 elements. The wire medium-based solution reveals an effective way for reducing the blind-spot in a wide bandwidth, while the metaradome has been found less suitable for the same purpose.
\end{abstract}

Key words: Phased array, Scan blindness, Metasurface, Metamaterial, Wire medium.

\section{Introduction}

Phased-array antennas are widely used in radar applications due to their beam steering capability. For this reason, this type of antennas has been the backbone of multiple radar systems since this topic took off in the $60 \mathrm{~s}$ of last century $[1,2]$. The radiation pattern of these antennas is electronically controlled by properly tuning the phase and/or amplitude of each element. Phased-array antennas are crucial devices in the development of new systems for many military and civil applications.

Scan blindness is a well-known artifact present in large phased array antennas with large beam-scan ranges. It is manifested as a strong mismatch of the active impedance when the scan reaches a certain angle. This phenomenon has two different main causes, both due to the periodic nature of the geometry and to the linear phasing. The first one is the most common and happens when the first higher order Floquet (Bloch) mode of the periodic structure transits from evanescent

*e-mail: miguel.beruete@unavarra.es to propagating regime causing a strong interaction among the elements [3]. Hence, this first mechanism will be called in this document as "onset of grating lobe". It occurs only if the spacing is larger than half free-space wavelengths and is the most critical for largely spaced array elements. A second cause is due to a mode interaction (namely, a synchronism of phase velocities) between a higher order Floquet mode and an eigenmode supported by the periodic boundary conditions. This second type is more accused for dense arrays, and its interpretation dates back to the $60 \mathrm{~s}$ of last century, thanks to the seminal papers of Oliner et al. [4], who also showed the relationship with Wood's anomalies [5]. In the $80 \mathrm{~s}$ Pozar and Schaubert [6] studied the simplified case of an array of printed dipoles. They demonstrated that scan blindness occurs when the first evanescent Floquet mode and the dominant surface (or substrate) wave propagation constants coincide.

The most straightforward solution to avoid blinds spots due to grating lobes is to reduce the coupling between elements. However, there are cases where the distance between elements is a design constraint that cannot be overridden. In those cases, other strategies such as the inclusion of artificial structures 
have to be considered. In particular, wide angle impedance matching (WAIM) techniques using conventional dielectrics have been used in the past for matching an antenna over the entire angular scanning domain [7]. However, these conventional WAIMs do not affect significantly the blind-spot.

In the last years, there has been a growing activity in the research of artificial structures such as metamaterials to control the propagation of electromagnetic waves and, in particular, deal with the effects derived from surface wave excitation, propagation and diffraction. Metamaterials are usually defined as manmade structures exhibiting electromagnetic properties that are not available in nature. They have been proposed for multiple antenna applications such as enhanced gain superstrates [8], to reduce the coupling between antenna elements [9], or to enhance the efficiency of electrically small antennas [10] to name a few. More recently, metamaterial inspired anisotropic WAIMs have been theoretically proposed for an array of open-ended waveguides antennas [11] and for improving the performance at large scanning angles for a phased array antenna [12]. In both cases, the WAIM was designed in absence of extreme impedance conditions (grating lobes) which permits the matching for the targeted scanning angles while keeping other directions and cardinal planes unaltered. Recently, an innovative tool for synthesis of multifrequency, multilayer and uniaxially anisotropic WAIMs structures for waveguide-fed phased array antennas has been presented [13].

In this work, metamaterial concepts are used to improve the radiation characteristics of a phased array antenna system for a radar application. The main objective in this particular framework is to enhance the antenna performance by mitigating the blind-spot effect working at the radome level and without changing the distance between antenna elements. The goal of this work is to extend the angular scanning domain up to $\pm 50^{\circ}$ while preserving an operational bandwidth of at least $1 \mathrm{GHz}$ around the central frequency of $10 \mathrm{GHz}$ (fractional bandwidth of $10 \%$ ). To this end, two different strategies have been followed. The first approach is focused on reducing coupling elements by using a metasurface-based radome (metaradome) based on a matrix of metallic wires, so-called bed of nails structure [14]. This approach was particularly inspired by [15]. However, the final wire medium metaradome (WMM) is adapted to the fabrication constrains given by the antenna architecture $[16,17]$.

In the second approach, the focus is put on impedance and matching concepts. A solution similar to the one proposed in $[11,12]$ is considered here, with the additional difficulty that the onset of a grating lobe lies within our region of interest introducing a pronounced impedance mismatch between the antenna and the free-space. In our case of study, the presence of grating lobes gives rise to a very steep impedance profile hindering the matching within a wide frequency band and operation angle. The adopted solution is a WAIM metaradome (WAIMM) based on a periodic Jerusalem cross structure [18].

The paper is organized as follows: Section 2 gives a description of the prototype antenna and the blind-spot problem. In Section 3 a description of the design process and simulation results of both solutions are provided. Section 4 presents the measurement results. Finally, Section 5 summarizes the content of the paper and provides some closing conclusions.

\section{Description of the problem}

\subsection{Prototype antenna and blind spot discussion}

The phased-array antenna under study is an array composed of one hundred patch elements arranged in a $5 \times 20$ rectangular topology (see Figure 1). The unit cell is a square patch antenna of side $a=7.8 \mathrm{~mm}$ printed on a Rogers RO4003 substrate with permittivity $\varepsilon_{r}=3.55$ and height $h_{\text {subs } 1}=0.81 \mathrm{~mm}$ and fed with a coaxial probe. This single element is embedded in a metallic cavity closed on the top by a parasitic square patch slightly smaller than the radiating element with side $b=7.5 \mathrm{~mm}$ printed on another Rogers RO4003 dielectric substrate with height $h_{\text {subs } 2}=0.51 \mathrm{~mm}$. The cavity height, i.e. distance between patch and parasitic elements, is $H=3 \mathrm{~mm}$. The interelement spacing is $D=19 \mathrm{~mm}$. Figure 2 shows the reflection coefficient of a single element and infinite array antenna simulated with the commercial solver CST Microwave Studio ${ }^{\mathrm{TM}}$ along with a schematic model and E-plane radiation pattern at $10 \mathrm{GHz}$ of the single antenna. As shown, the operational bandwidth of the isolated antenna is about $2 \mathrm{GHz}$. However, for the infinite array the bandwidth is reduced due to coupling effects between elements.

As mentioned in the introduction, scan blindness may arise in phased-array configurations due to the interaction between grating modes and surface waves. Consequently, a blind spot can appear in the radiation pattern at the directions and frequencies where these interactions occur. In the studied case, the blind spot coincides with the grating lobe emergence which is determined by the following well-known equation:

$$
\frac{D}{\lambda_{0}}=\frac{1}{\sin \theta_{\mathrm{GL}}+\sin \theta_{0}}
$$

where $D$ is the distance between elements, $\lambda_{0}$ is the freespace wavelength at the operation frequency, $\theta_{\mathrm{GL}}$ is the angle of the first grating lobe and $\theta_{0}$ is the scanning/incidence angle [19]. Particularizing (1) for $\theta_{\mathrm{GL}}=90^{\circ}$ (grating lobe radiation at grazing angle) the onset of the first grating lobe condition is:

$$
\frac{D}{\lambda_{0}}=\frac{1}{1+\sin \theta_{0}}
$$

Whenever there is excitation of a grating order there is energy associated to it. So in this particular case, part of the total energy is transferred from the 0 -order (we could say also main beam) to the 1-order. In order to see the connection between the blind spot and the grating lobe, infinite array simulation results of the reflection coefficient $(|\mathrm{S} 11|)$ and transmitted energy $(|\mathrm{S} 2(m), 1|)$ for $m=0$ and $m=-1$ grating orders can be found in $[16,17]$. At the onset of the grating lobe (around $34.5^{\circ}$ ) it is observed that the reflection increases up to about $0 \mathrm{~dB}$ (the calculation with Eq. (3) gives $35.37^{\circ}$ ) while a strong dip appears in the transmission of the $m=0$ grating order. Additionally, it can be seen that beyond resonance, the $m=-1$ order transmission rises, i.e. the grating lobe enters into real space and starts to radiate.

The $5 \times 20$ phased-array prototype antenna was fabricated and measured. From the measured coupling coefficients it is 


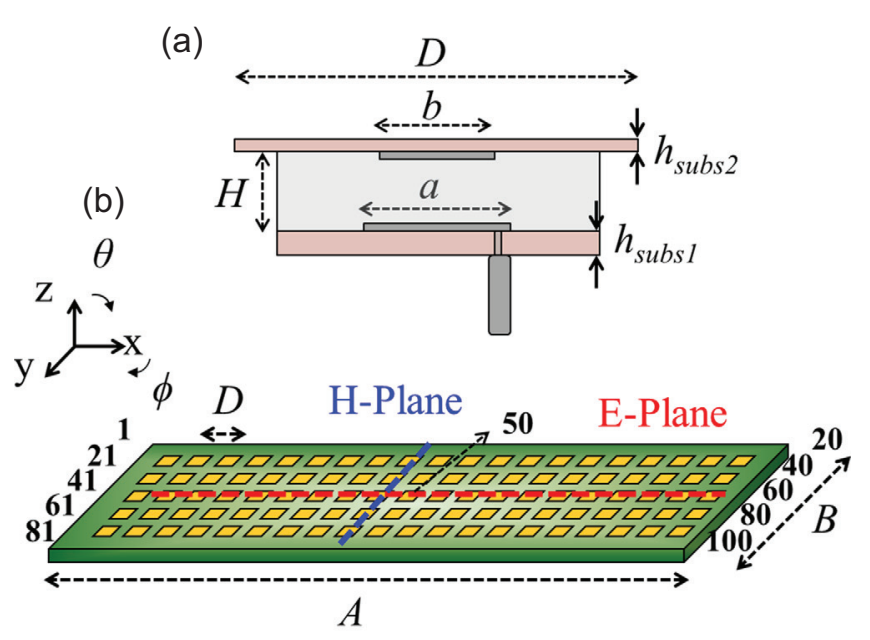

(c)

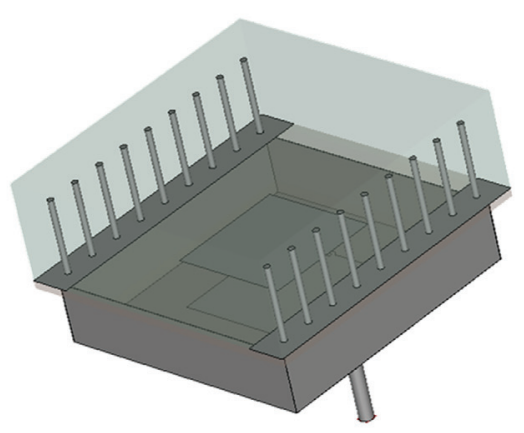

(d)

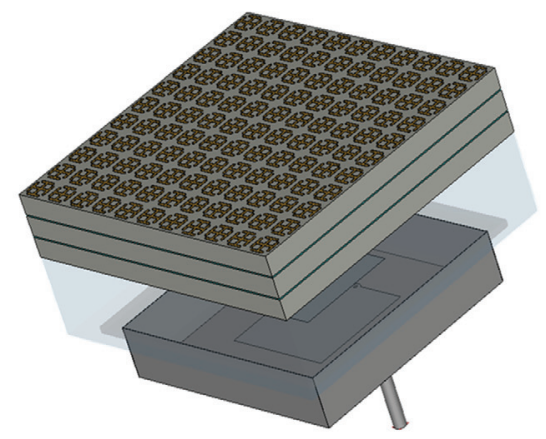

Figure 1. Schematics showing the original antenna element and the solutions developed. (a) Unit cell cross section with parameters. (b) $5 \times 20$ array schematic with E- and H-planes outlined. Numbers at right and left of the array denote the element number in the array (e.g. 50 is the central element). Dimensions: $A=400 \mathrm{~mm}$, $B=115 \mathrm{~mm}, \quad D=19 \mathrm{~mm}, \quad a=7.8 \mathrm{~mm} \quad$ and $\quad b=7.5 \mathrm{~mm}$, $h_{\text {subs } 1}=0.81 \mathrm{~mm}$ and $h_{\text {subs2 }}=0.51 \mathrm{~mm}$. (c) Schematic unit cell of wire medium-based metaradome. (d) Schematic unit cell of Jerusalem cross-based metaradome.

possible to obtain the active reflection coefficient by applying the following equation:

$$
R_{n}(\theta, \phi)=\sum_{i=1}^{N} S_{n i} e^{-j \varphi_{i}}
$$

where $\theta$ and $\phi$ are the elevation and azimuth angle respectively, $S_{n i}$ are the measured coupling coefficients and $\varphi_{i}$ is the phase difference between $n$ and $i$ elements, see schematic in the inset of Figure 3. This figure shows the magnitude of

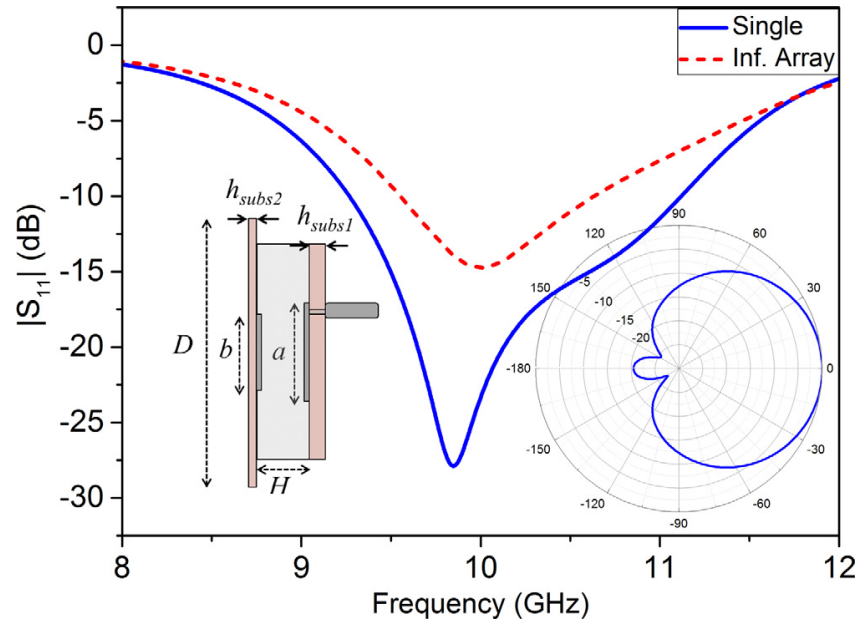

Figure 2. Simulated reflection coefficient of single element antenna. Insets: Antenna schematic and E-plane radiation pattern at $10 \mathrm{GHz}$.

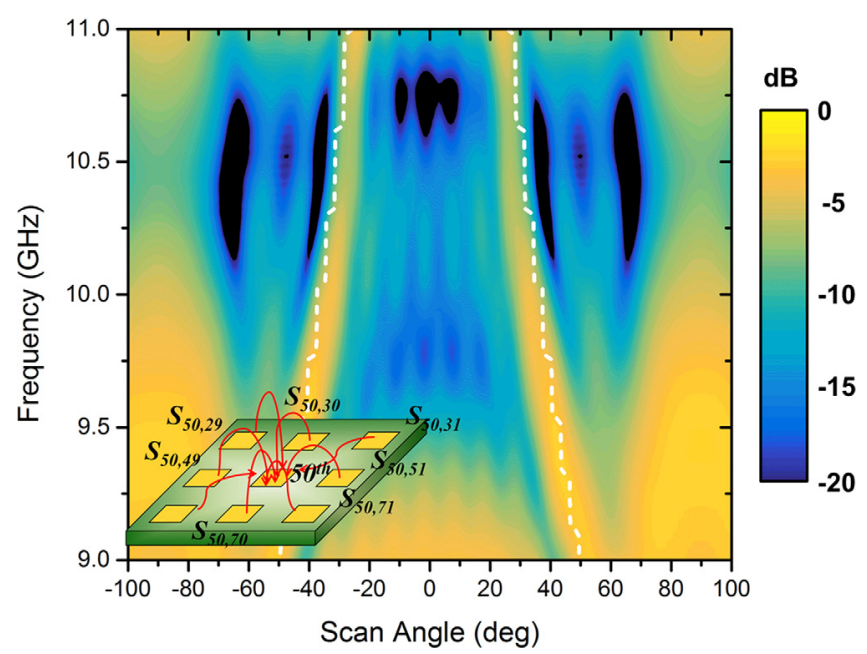

Figure 3. E-plane scan reflection coefficient for the 50th element over frequency and angle of incidence (scale in decibels). Dashed white line corresponds to theoretical result of the onset of the first grating lobe. Inset: Schematic of coupling coefficients contribution for one element active reflection coefficient.

the active reflection coefficient for the 50th (central element) over frequency and scanning angle (E-plane). The operational bandwidth of the fabricated antenna (defined as the frequency range where the active reflection coefficient is below $-10 \mathrm{~dB}$ ) extends from $9.5 \mathrm{GHz}$ up to $11 \mathrm{GHz}$ approximately at broadside scanning. The white-dashed line marks the onset of the grating lobe, which coincides with a high active reflection coefficient magnitude introducing a blind spot in the radiation pattern and limiting the scanning angle range.

When dealing with infinite arrays the onset of a grating lobe leads to a severe blind spot in the radiation pattern. In terms of scan impedance this condition leads to a singularity going to infinity and therefore resulting in an extreme impedance mismatching [1]. However, for finite arrays this impedance singularity is less sharp leading to a reduction of 


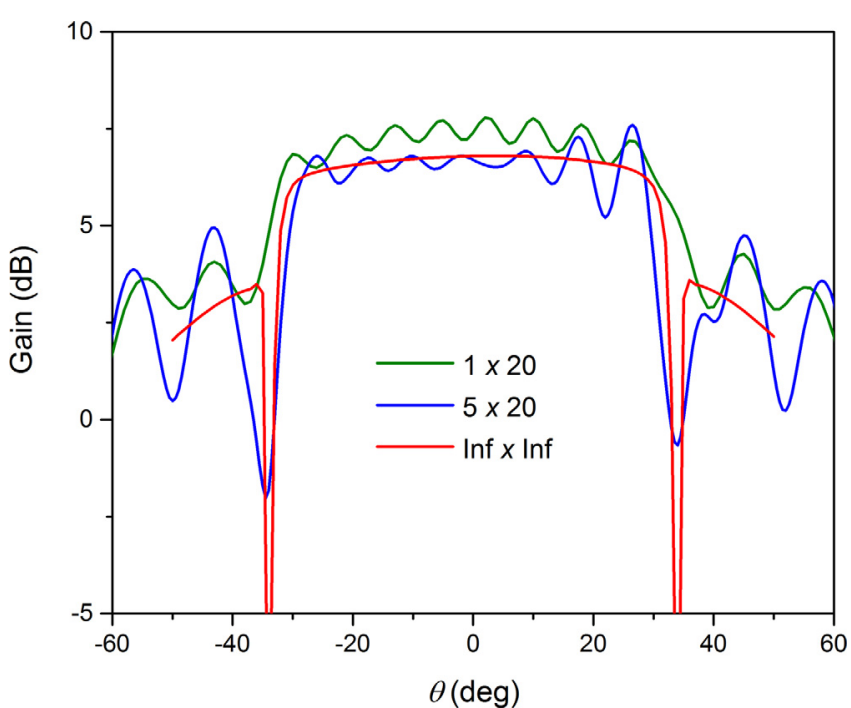

Figure 4. Element radiation pattern of a 1 row of 20 elements array (element \#10), a 5 rows of 20 elements array (element \#50) and an infinite array.

radiated power rather than a deep null, as demonstrated in [16, 17]. Figure 4 is reproduced here for completeness.

\section{Metaradome design}

\subsection{Wire medium metaradome}

Given the wide frequency bandwidth requirement, a stop band effect is preferred rather than single narrowband resonant effect. Thus, the so-called structure bed of nails [14] seems to be an appropriate choice since it exhibits filtering properties and its implementation is compatible with the layout of the antenna array. This metamaterial consists of periodic metallic pins connected to a metallic plane. An homogenization model has been proposed in the literature $[20,21]$ able to give the mode characteristics of the metamaterial and the $k_{x}$ wavenumber evolution in relation to the geometrical variables. This homogenization model gives two useful indications for the design of wire medium structures:

(i) The pin length $L$ determines the stop band frequency as follows:

$$
f \geq \frac{c}{4 L \sqrt{\varepsilon_{r}}}
$$

(ii) The increase of the effective plasma frequency leads to a higher value of the tangent wave vector component $\left|k_{x}\right|$ for frequencies close to the stop band limit. The plasma frequency can be estimated by using [22]:

$$
f_{p}=\frac{c}{a \sqrt{2 \pi \varepsilon_{r}}}\left(\ln \left(\frac{a}{2 \pi r}\right)+0.525\right)^{-1 / 2}
$$

where $r$ is the radius of the wires and $a$ the inter-wire spacing.

The results of the tangential wave vector component (both real and imaginary parts) are shown in Figure 5. The gray

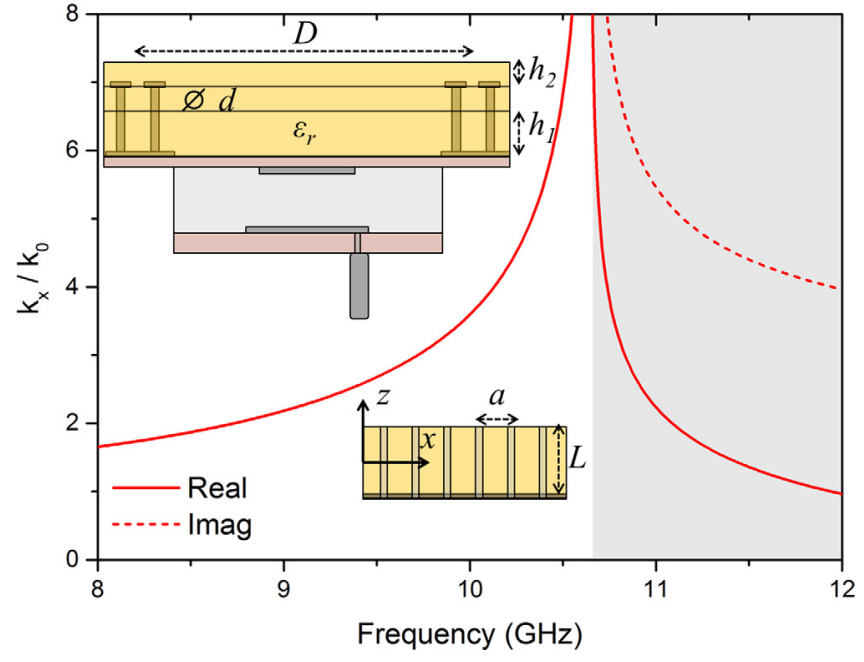

Figure 5. Frequency evolution of the $x$-component of the real (solid line) and imaginary part (dashed line) wave vector of a TM surface wave which propagates along a fakir's bed of nails with length $L=4.75 \mathrm{~mm}$, separation distance of $a=2.38 \mathrm{~mm}$ and host dielectric permittivity of $\varepsilon_{r}=2.2$. Insets: Bottom: Fakir's bed of nails analyzed. Top: Metaradome placed on top of one antenna element. Dimensions: $D=19 \mathrm{~mm} ; d=0.4 \mathrm{~mm} ; h_{1}=3.175 \mathrm{~mm}$; $h_{2}=1.575 \mathrm{~mm}$.

shaded area denotes the region where improper or evanescent waves are present. The integration of the bed of nails metamaterial structure in the array was done by taking into account a number of constraints. First, the available space between the radiating elements is limited. Indeed, the array periodicity $(19 \mathrm{~mm})$ and the size of the cavity $(12 \mathrm{~mm})$ leave a gap of only $7 \mathrm{~mm}$ where the artificial media can be inserted. Secondly, the structure had to be placed over the layer supporting the parasitic patch of the radiating element to keep the host antenna with its original configuration. This means that the metal strip connecting the wires is not in contact with the ground plane, which guides the surface waves responsible for the blind spot phenomenon. Consequently, theoretical formulas cannot be used to accurately predict the stop band frequency and a complete numerical optimization must be carried out.The optimal solution was found taking into account the previously described constraints, see top inset in Figure 5. It consists of a double row of metallic wires terminated with a metallic disk and embedded in a host dielectric block with permittivity $\varepsilon_{r}=2.2$. For the practical implementation, several dielectric layers were needed. The wires were implemented as metal via in the first two layers whereas the third layer was used for impedance matching of the radiating element to the free space. The structure was placed perpendicular to the direction of propagation of the surface wave (i.e. perpendicular to $x$ ). The effect of the metaradome is that the energy of the surface waves unable to propagate due to the stopband characteristic of the wire structure, will contribute to the zeroth grating order.

\subsection{WAIM Jerusalem cross-based metaradome}

Alternatively, the onset of a grating lobe can be seen as a great impedance mismatch between the antenna and the 
(a)

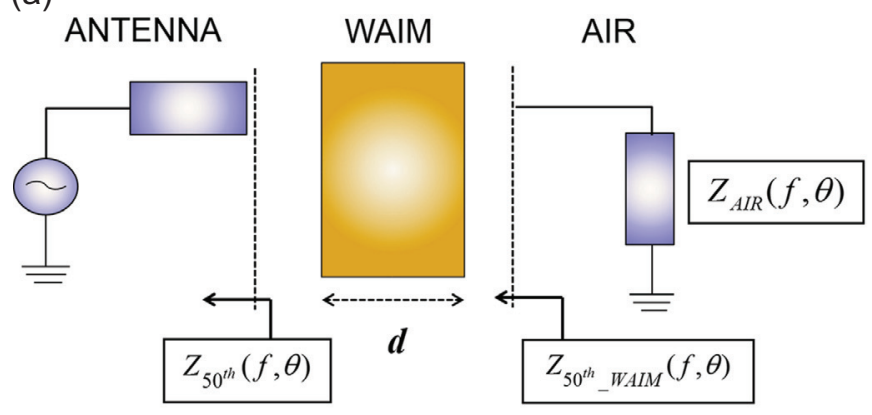

(b)

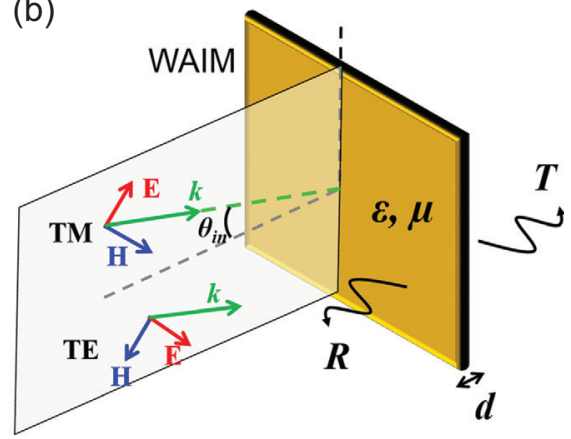

Figure 6. (a) Equivalent circuit block diagram of equivalent transmission line problem comprising the antenna and finite WAIM slab system embedded in air. (b) TM and TE oblique incidence of a plane wave on an infinite dielectric slab with finite thickness $d$ and constitutive parameters $\varepsilon$ and $\mu$.

free-space. Then, a very abrupt impedance profile against scanning angle appears and hence, the matching for all the angles and over a wide frequency range is highly challenging. The scan antenna impedance can be evaluated from the scan reflection coefficient as follows:

$$
Z_{n}(\theta, \phi)=Z_{0} \frac{1+R_{n}(\theta, \phi)}{1-R_{n}(\theta, \phi)}
$$

where $Z_{0}$ is the free-space impedance and $R_{n}$ the $n$th scan element reflection coefficient.

An anisotropic slab is proposed here for the WAIM metaradome since it provides more degrees of freedom. The first step consists of finding a homogenous anisotropic slab with ad-hoc constitutive parameters and hence, the required impedance behavior. To this end, classical transmission line theoretical concepts and analytical equations for oblique incidence over anisotropic finite thickness slabs can be applied [23]. Figure 6a shows a block diagram where a finite WAIM slab of thickness $d$ is designed to match the active impedance of element 50th. Matching occurs when $Z_{50 \text { th }}(f, \theta)=Z_{\mathrm{AIR}}^{*}(f, \theta)$ with $f$ and $\theta$ being the frequency and scanning angle respectively. To get a complete characterization, both transverse electric (TE) and transverse magnetic (TM) polarizations must be considered (see Figure 6b).

This way, a rapid analytic tool can be easily embedded into an optimization process which enables an effective parameter search for a specific result target. For this purpose, the optimization toolbox of the commercial software MATLAB ${ }^{\circledR}$, which includes different optimization algorithms such as direct search (DS), genetic algorithm (GA) or simulated annealing (SA), was used for the optimization routines. For this particular problem, the SA algorithm was able to reach an optimal solution. Therefore, an optimization routine based on this algorithm was launched obtaining a good WAIM candidate in terms of impedance matching for the 50th element at $10 \mathrm{GHz}$ as a result. To this end, $R_{50}$ was forced to be below $R_{\mathrm{ref}}=-10 \mathrm{~dB}$ for the entire angular range (up to $\theta_{\max }=50^{\circ}$ ). The cost function, $\Theta(\bar{\varepsilon}, \bar{\mu}, d)$ was calculated as the minimum square error between $R_{\text {ref }}$ and $R_{50}$ (for the $\theta$ cases where $R_{50}>R_{\text {ref }}$ ) as follows:

$$
\Theta(\bar{\varepsilon}, \bar{\mu}, d)=\sum_{\theta=0}^{\theta_{\max }}\left(R_{\text {ref }}-R_{50}(\theta)\right)^{2}
$$

where $\bar{\varepsilon}$ and $\bar{\mu}$ are the permittivity and permeability tensors and $d$ the slab thickness. Evidently the goal is achieved when $\Theta=0$. The WAIM obtained is not necessarily optimal for different elements and/or frequencies. Besides, the optimization was performed uniquely for the E-plane, which is where the blind-spot artifact manifests more abruptly. Therefore, the H-plane could be negatively affected when including the WAIM slab.

Figure 7 shows the scan reflection coefficient in the presence of a homogenous WAIM with the following constitutive parameters: $\varepsilon_{x x}=\varepsilon_{y y}=6.8, \varepsilon_{z z}=4, \mu_{x x}=\mu_{y y}=\mu_{z z}=1$. The total thickness of the slab is $d=4.812 \mathrm{~mm}$. These values were constrained in terms of fabrication feasibility and commercial substrates availability. Therefore the degrees of freedom of the optimization routine where limited to three: $\varepsilon_{x x}$ (or $\left.\varepsilon_{y y}\right), \varepsilon_{z z}$ and $d$. As it can be seen in Figure 7 the matching for the central frequency improves up to levels below $-10 \mathrm{~dB}$ for the total scanning view. On the other hand, the performance at other regions of the frequency/angle area is deprecated when compared with the response without the anisotropic WAIM (see Figure 3). It can also be observed that total reflection arises for the entire frequency bandwidth at scanning angles around $80^{\circ}$. Figure 8 provides the scan impedance (real and imaginary part) for the central element at the complete angular range for the array antenna without any structure on top and with an anisotropic homogenous slab acting as a WAIM. Again, in line with previous discussion, the blind spot is identified as a singularity of the scanning impedance leading to a huge and sharp mismatch. When including the homogenous WAIM the impedance is modified to values close to the free space at this blind spot direction. However, the scan impedance varies also at other angles where the antenna without WAIM was well-matched, leading to a possible reduction of the performance at those directions.

The synthesis of a metamaterial exhibiting the aforementioned constitutive was conducted. For practical issues during the fabrication process, the WAIM design was simplified in order to get a feasible metamaterial particle. The final outcome results were limited to WAIMs exhibiting uniquely electric response. This can be synthesized with a purely electric resonator such as a Jerusalem cross. This particle, among others, has been widely used in frequency selective surfaces (FSS) applications. The major difference here is that the 


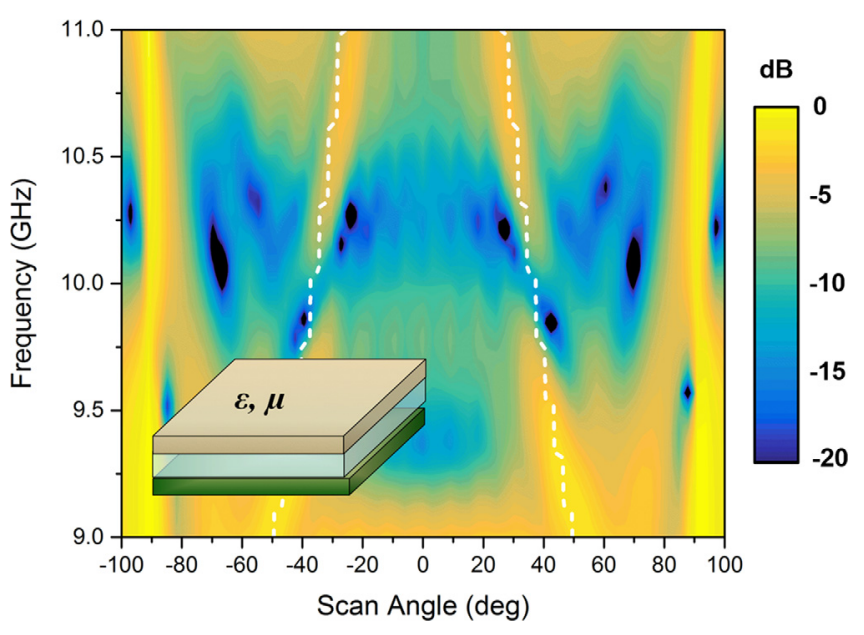

Figure 7. E-plane scan reflection coefficient for the central element vs. frequency and angle of incidence in the presence of the homogenous WAIM slab (scale is in decibels). Dashed white lines is the theoretical onset of the first grating lobe.

operation frequency is much below the self-resonance of the particle.

In order to get the specific cross shape that delivers the desired $\varepsilon$ and $\mu$, a retrieval process [24] was iteratively launched modifying the physical geometry of the particle at each step. To this end, the full wave simulator CST Microwave Studio was used for simulating the unit cell in a Floquet boundary condition scheme. Then, the scattering parameters were extracted and introduced in the retrieval method described in [25]. Once the retrieved $\varepsilon$ and $\mu$ were identical to the proposed values for the homogenous WAIM the metaradome design was finished. Figure 9 shows the retrieved $\varepsilon$ and $\mu$ over frequency. It can be seen that at $10 \mathrm{GHz}$ the values correspond with those obtained in the previous step. However, there is certain dispersion in the frequency response. The fabricated metaradome is a multilayer structure consisting of three Rogers 4003 printed substrate layers glued by Rogers $4350 \mathrm{~B}$ prepreg films. The geometry and dimensions of the unit cell are shown in Figure 9 insets.

For practical reasons, a foam substrate was placed between the antenna and the radome. This way the metaradome can be conveniently attached to the antenna top surface and the gap between both structures can be filled with a material with relative permittivity close to the free-space one.

\section{Experimental results}

The experimental demonstration of both proposals, WMM and WAIMM, were conducted in parallel. To this end a pair of antenna prototypes were also tested with each metaradome solution. In both cases, the measurements were conducted in anechoic chambers and each element was connected to a VNA while the others were terminated with $50 \Omega$ loads. With this measurement technique that includes the array environment of each radiating element, the complete antenna

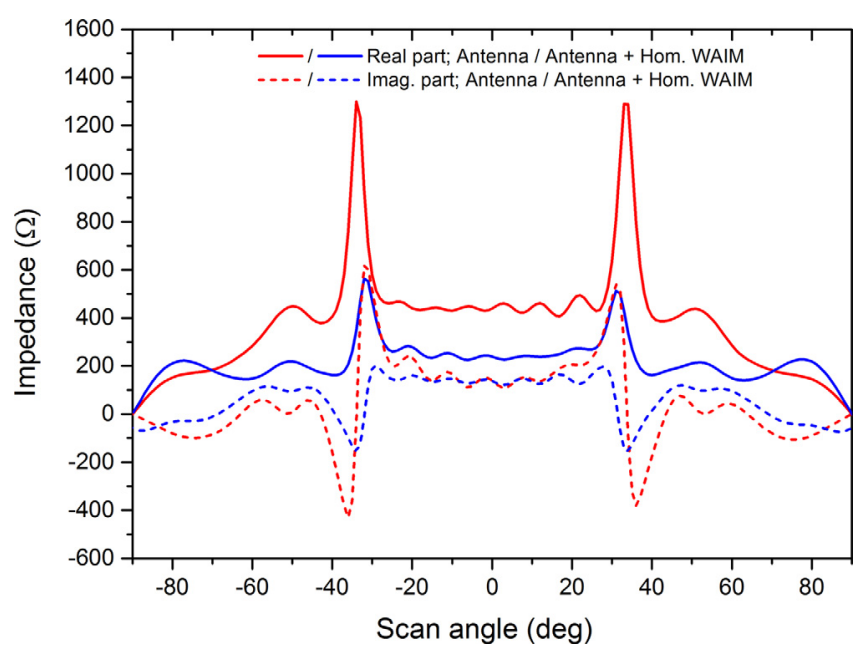

Figure 8. E-plane scan impedance for the 50th element versus angle of incidence for the infinitely replicated prototype antenna without (solid line) and with an homogenous anisotropic WAIM on top (dashed line). Red color stands for the real part while blue color line corresponds to the imaginary part.

pattern could be calculated by summing the 100 radiation patterns.

Figure 10 shows the normalized element pattern for the central element of the array antenna without and with the metaradome proposals at different frequencies. Blue color lines are linked to the first prototype (dashed line) and wire medium metaradome (solid line) and red color lines correspond to the second prototype (dashed line) and the WAIMM (solid line). As it can be observed, the blind spot can be clearly identified by the two low-level symmetrical lines across the angularfrequency space. When the WMM is added, the blind directions are significantly mitigated for a wide band operation. Furthermore, the gain is slightly increased at frequencies over $10 \mathrm{GHz}$. On the other hand, the performance of the WAIMM is less effective for a wide band operation. Indeed, a small reduction of the grating lobe is achieved by the central frequency of $10 \mathrm{GHz}$ and close to it, $9.9 \mathrm{GHz}$ (see Figures 10a and 10d). However, the blind spot is split into two dips of reduced level. This is a consequence of the narrowband nature of this solution. The WAIMM was optimized to the central frequency and the blind spot angle but it might not be effectively matched to the neighboring frequencies/angles. In addition, due to the very abrupt profile of the impedance nearby the blind spot angle, little differences between the predicted and the actual impedance can lead to strong disagreement between simulation and measurements. It must also be taken into account that the transmission line approach followed here is a first order approximation. Therefore, the possible coupling between the antenna and the WAIMM was not considered in the previous calculations. This could be the reason for the stronger ripple observed in the experimental radiation pattern.

Given the results obtained, the optimal solution for a wide band and effective blind spot mitigation is the WMM. The WAIMM here is not optimal given that the scan impedance variation with frequency and angle, and also between 

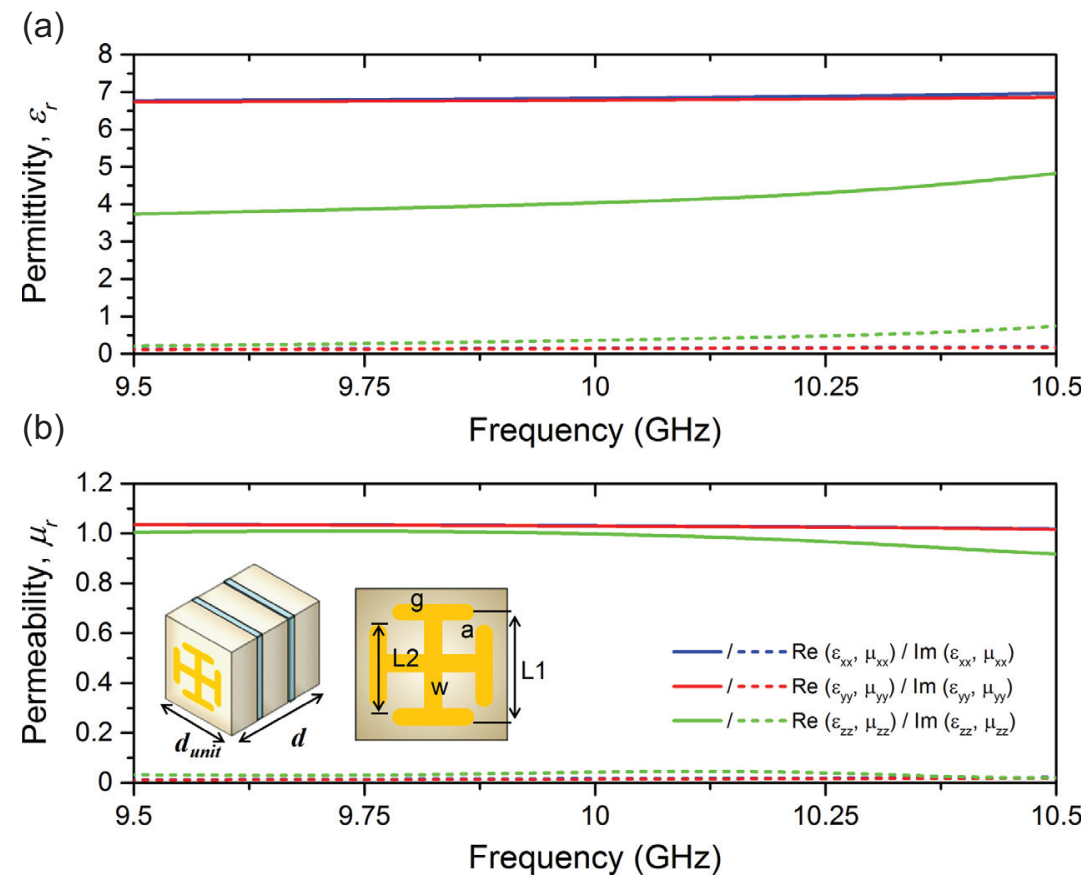

Figure 9. Retrieved permittivity (a) and permeability (b) of the simulated WAIM. Inset: Side and front view of metamaterial unit cell: $d_{\text {unit }}=2 \mathrm{~mm}, d=4.812 \mathrm{~mm}, L_{1}=1.6 \mathrm{~mm}, L_{2}=1.1 \mathrm{~mm}, a=0.1 \mathrm{~mm}, g=0.2 \mathrm{~mm}$ and $w=0.2 \mathrm{~mm}$.

(a)

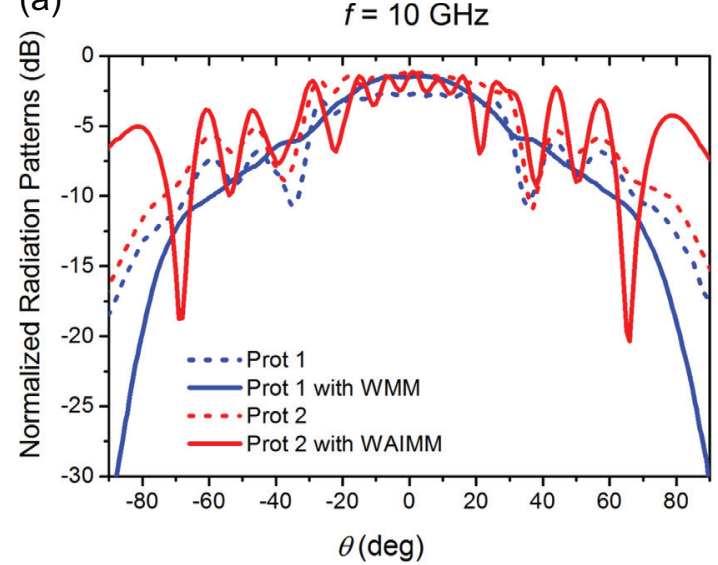

(c)

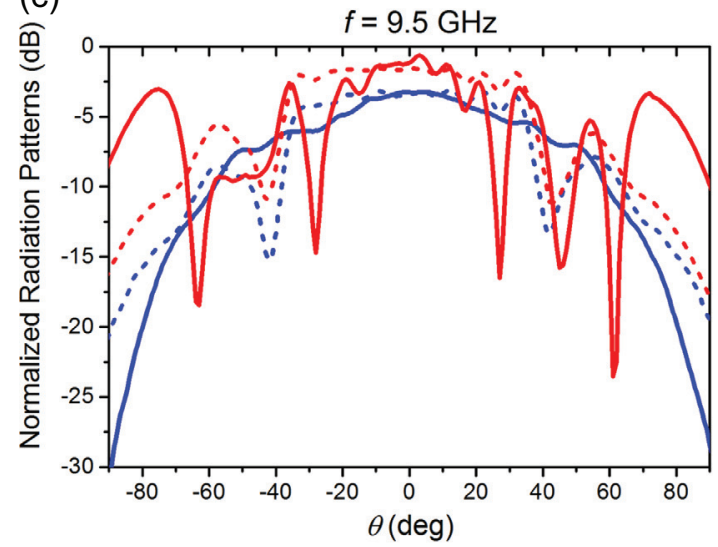

(b)

$f=10.5 \mathrm{GHz}$

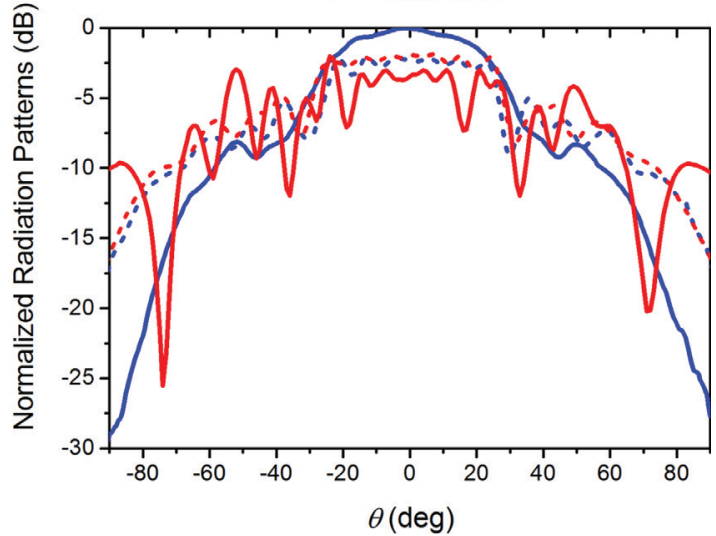

(d)

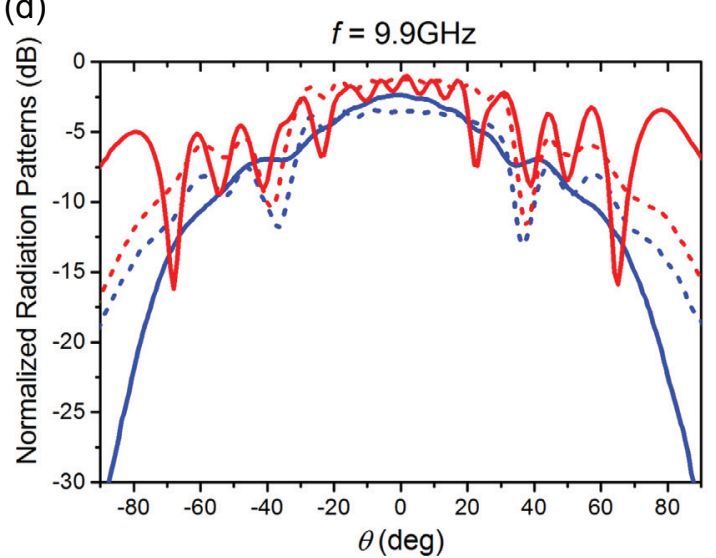

Figure 10. Experimental E-plane central element radiation pattern at different frequencies. Blue color line corresponds to the first prototype (wire medium solution) with (solid) and without (dashed) metaradome. Red color line corresponds to the first prototype (Jerusalem cross based solution) with (solid) and without (dashed) metaradome. 
array elements, is very steep due to the presence of the onset of a grating lobe. Nevertheless, if a smoother impedance profile is provided (e.g. after eliminating the grating lobe condition with the WMM presented) this WAIMM could enhance the scanning field of view by improving the matching between the antenna and the free-space.

\section{Conclusions}

In this manuscript two different metamaterial based radome solutions for a phased-array antenna in a radar application have been presented. The main challenge of the design was to mitigate the blind-spot effect originated by the onset of a grating lobe. The connection of this grating lobe condition with surface wave excitation has been identified and observed in full wave numerical simulations.

A metaradome based on the so-called bed of nails structure has been demonstrated as a successful solution capable of improving the radiation characteristics of the original antenna. Radiation patterns for the E-plane prove that the blind-spot condition disappears or is highly mitigated. Moreover, grating lobes are significantly reduced. However, some drawbacks have been observed: a gain reduction for frequencies below $10 \mathrm{GHz}$ (3-5 dB at broadside) and slight performance degradation in the H-plane scanning.

A metaradome based on subwavelength Jerusalem-cross particles has been also designed. The semi-analytic process followed has been described. In this case, the metaradome is a WAIM-inspired structure. Again, the main obstacle is the extreme impedance mismatch because of the onset grating lobe appearance. Regarding the results obtained, it might not be the best approach for mitigating the blind spot over a wide frequency band and scanning view. Moreover, here the metaradome is uniform for the complete array dimensions. One might think about a gradient metaradome that would provide the proper matching needed at each element. Another option to provide wideband operation would be to run several optimization routines at different frequencies finding the most suitable meta-particle for each one and combining the particles conveniently in a periodic pattern.

All in all, the wire medium solution opens up a very interesting utility for metamaterials in the design of radomes for phased-array antennas where reducing the interelement spacing for avoiding grating lobes is not practical. Moreover, it could be potentially enhanced by combining it with a WAIM-based solution able to enhance the matching of the antenna given that then the extreme impedance condition has been previously mitigated.

Acknowledgements. In memoriam of our beloved friend Mario Sorolla, who was the driving force and source of inspiration for this work.

This work was supported by the METALESA project A-1089RT-GC that was coordinated by the European Defence Agency (EDA) and funded by 11 contributing Members (Cyprus, France, Germany, Greece, Hungary, Italy, Norway, Poland, Slovakia, Slovenia and Spain) in the framework of the Joint Investment Programme on Innovative Concepts and Emerging Technologies (JIP-ICET).

\section{References}

1. R.C. Hansen, Microwave Scanning Antennas. vol. 1, Apertures, Academic Press, New York and London, 1964.

2. L. Stark, Microwave theory of phased-array antennas: a review, Proc. IEEE 62 (1974) 1661-1701.

3. R.J. Mailloux, Phased array antenna handbook, Artech House, Boston, 2005.

4. G.H. Knittel, A. Hessel, A. Oliner, Element pattern nulls in phased arrays and their relation to guided waves, Proc. IEEE 56 (1968) 1822-1836.

5. A. Hessel, A.A. Oliner, A new theory of Wood's anomalies on optical gratings, Appl. Opt. 4 (1965) 1975-1297.

6. D. Pozar, D. Schaubert, Scan blindness in infinite phased arrays of printed dipoles, IEEE Trans. Antennas Propag. 32 (1984) 602-610.

7. E. Magill, H.A. Wheeler, Wide-angle impedance matching of a planar array antenna by a dielectric sheet, IEEE Trans. Antennas Propag. 14 (1966) 49-53.

8. H. Attia, L. Yousefi, M.M. Bait-Suwailam, M.S. Boybay, O.M. Ramahi, Enhanced-gain microstrip antenna using engineered magnetic superstrates, IEEE Antennas Wirel. Propag. Lett. 8 (2009) 1198-1201.

9. M.M. Bait-Suwailam, M.S. Boybay, O.M. Ramahi, Electromagnetic coupling reduction in high-profile monopole antennas using single-negative magnetic metamaterials for MIMO applications, IEEE Trans. Antennas Propag. 58 (2010) 2894-2902.

10. R.W. Ziolkowski, A. Erentok, Metamaterial-based efficient electrically small antennas, IEEE Trans. Antennas Propag. 54 (2006) 2113-2130.

11. S. Sajuyigbe, M. Ross, P. Geren, S.A. Cummer, M.H. Tanielian, D.R. Smith, Wide angle impedance matching metamaterials for waveguide-fed phased-array antennas, IET Microw. Antennas Propag. 4 (2010) 1063-1072.

12. F. Silvestri, P. Chiusolo, L. Cifola, R. Bolt, G. Gerini, Design of metamaterial based wide angle impedance matching layers for active phased arrays, Antennas and Propagation (EuCAP), 2015 9th European Conference on, Lisbon, 2015.

13. G. Oliveri, F. Viani, N. Anselmi, A. Massa, Synthesis of multilayer WAIM coatings for planar phased arrays within the system-by-design framework, IEEE Trans. Antennas Propag. 63 (2015) 2482-2496.

14. R.J. King, D.V. Thiel, K. Park, The synthesis of surface reactance using an artificial dielectric, IEEE Trans. Antennas Propag. 31 (1983) 471-476.

15. I.S. Nefedov, S.A. Tretyakov, J. Säily, X. Liangge, T. Mynttinen, M. Kaunisto, Application of wire media layers for coupling reduction in antenna arrays and microwave devices, Presented at the Loughborough Antennas and Propagation Conference, Loughborough, UK, 2007.

16. T. Crepin, C. Martel, B. Gabard, F. Boust, J.P. Martinaud, T. Dousset, P. Rodriguez-Ulibarri, M. Beruete, C. Loecker, T. Bertuch, J.A. Marcotegui, S. Maci, Blind Spot Mitigation in Phased Array Antenna using Metamaterials, 2014 International Radar Conference, Lille, 2014.

17. T. Crepin, C. Martel, B. Gabard, F. Boust, J.-P. Martinaud, T. Dousset, P. Rodríguez-Ulibarri, M. Beruete, C. Loecker, T. Bertuch, J.A. Marcotegui, S. Maci, Blind spot mitigation in phased array antenna using bed of nails, 5th International Conference on Metamaterials, Photonic Crystals and Plasmonics, META’ 14, Singapore, 2014. 
18. P. Rodríguez-Ulibarri, M. Beruete, F. Falcone, T. Crépin, C. Martel, F. Boust, C. Loecker, K. Herbert, C. Salzburg, T. Bertuch, J.-P. Martinaud, T. Dousset, J.A. Marcotegui, Metaradome for blind spot mitigation in phased-array antennas, 8th European Conference on Antennas and Propagation, EuCAP, The Hague, 2014.

19. B.A. Munk, Frequency selective surfaces: theory and design, Wiley-Interscience, USA, 2005.

20. M.G. Silveirinha, C.A. Fernandes, J.R. Costa, electromagnetic characterization of textured surfaces formed by metallic pins, IEEE Trans. Antennas Propag. 56 (2008) 405-415.

21. A.B. Yakovlev, M.G. Silveirinha, O. Luukkonen, C.R. Simovski, I.S. Nefedov, S.A. Tretyakov, Characterization of surface-wave and leaky-wave propagation on wire-medium slabs and mushroom structures based on local and nonlocal homogenization models, IEEE Trans. Microw. Theory Tech. 57 (2009) 2700-2714.
22. S.A. Tretyakov, Analytical modeling in applied electromagnetics, Artech House, Boston, 2003.

23. R.E. Collin, Field Theory of Guided Waves (IEEE Press Series on Electromagnetic Wave Theory). 2nd ed., Wiley-IEEE Press, New York, 1991.

24. D.R. Smith, D.C. Vier, T. Koschny, C.M. Soukoulis, Electromagnetic parameter retrieval from inhomogeneous metamaterials, Phys. Rev. E 71 (2005) 036617.

25. Z.H. Jiang, J.A. Bossard, X. Wang, D.H. Werner, Synthesizing metamaterials with angularly independent effective medium properties based on an anisotropic parameter retrieval technique coupled with a genetic algorithm, J. Appl. Phys. 109 (2011) 013515.

Cite this article as: Rodríguez-Ulibarri P, Crépin T, Martel C, Boust F, Falcone F, Loecker C, Herbertz K, Bertuch T, Dousset T, Martinaud J-P, Maci S, Marcotegui JA \& Beruete M: Experimental demonstration of metamaterials application for mitigating scan blindness in phased array antennas. EPJ Appl. Metamat. 2016, 3, 9. 